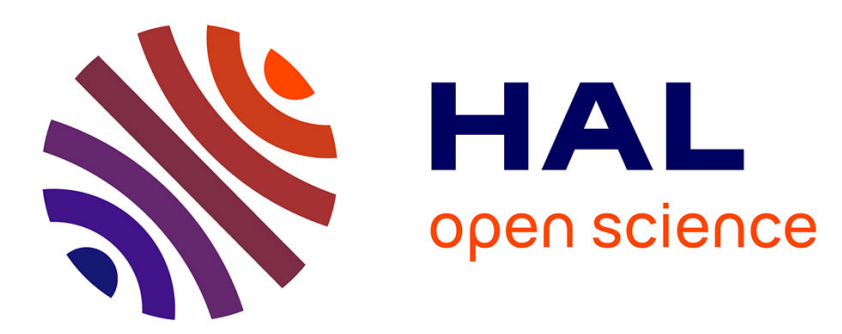

\title{
L'idéologie aliéniste du Dr Morel : christianisme social et médecine sociale, milieu et dégénérescence, psychiatrie et régénération. Partie II
}

F. Carbonel

\section{- To cite this version:}

F. Carbonel. L'idéologie aliéniste du Dr Morel : christianisme social et médecine sociale, milieu et dégénérescence, psychiatrie et régénération. Partie II. Annales Médico-Psychologiques, Revue Psychiatrique, 2010, 168 (9), pp.672. 10.1016/j.amp.2009.11.006 . hal-00690275

\section{HAL Id: hal-00690275 \\ https://hal.science/hal-00690275}

Submitted on 23 Apr 2012

HAL is a multi-disciplinary open access archive for the deposit and dissemination of scientific research documents, whether they are published or not. The documents may come from teaching and research institutions in France or abroad, or from public or private research centers.
L'archive ouverte pluridisciplinaire HAL, est destinée au dépôt et à la diffusion de documents scientifiques de niveau recherche, publiés ou non, émanant des établissements d'enseignement et de recherche français ou étrangers, des laboratoires publics ou privés. 


\section{Accepted Manuscript}

Title: L'idéologie aliéniste du Dr Morel : christianisme social et médecine sociale, milieu et dégénérescence, psychiatrie et régénération. Partie II

Author: F. Carbonel

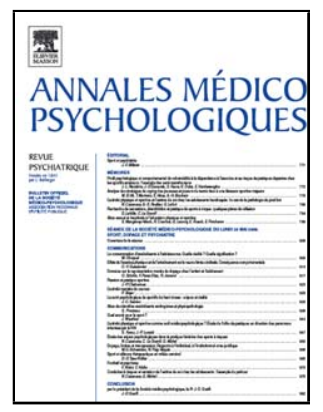

PII: $\quad$ S0003-4487(10)00246-5

DOI: $\quad$ doi:10.1016/j.amp.2009.11.006

Reference: $\quad$ AMEPSY 1215

To appear in: $\quad$ Annales Médico-Psychologiques

Received date: $\quad$ 15-6-2009

Accepted date: $\quad$ 7-11-2009

Please cite this article as: Carbonel F, L'idéologie aliéniste du Dr Morel : christianisme social et médecine sociale, milieu et dégénérescence, psychiatrie et régénération. Partie II, Annales medio-psychologiques (2010), doi:10.1016/j.amp.2009.11.006

This is a PDF file of an unedited manuscript that has been accepted for publication. As a service to our customers we are providing this early version of the manuscript. The manuscript will undergo copyediting, typesetting, and review of the resulting proof before it is published in its final form. Please note that during the production process errors may be discovered which could affect the content, and all legal disclaimers that apply to the journal pertain. 

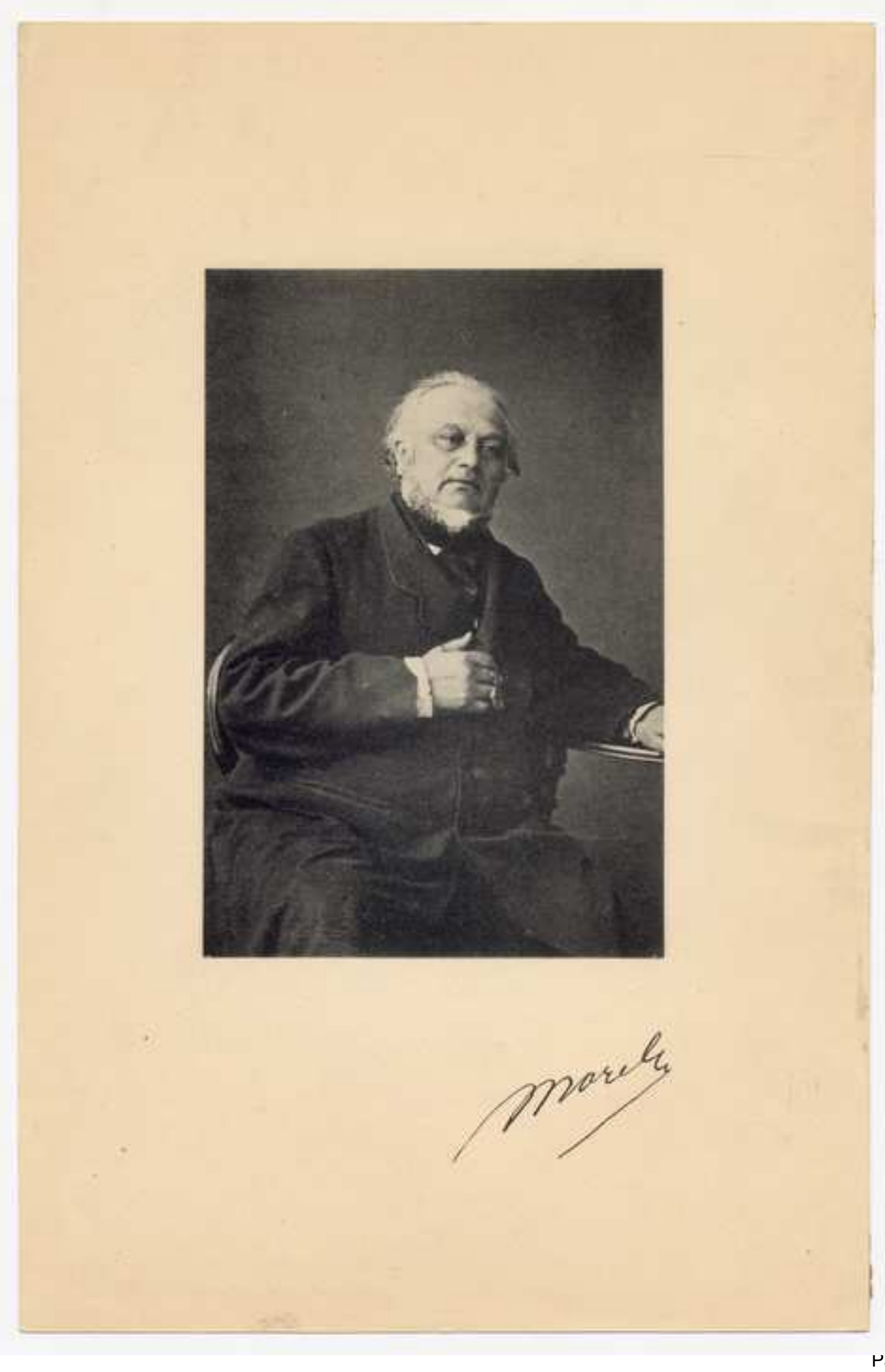

rage 1 of 19

.

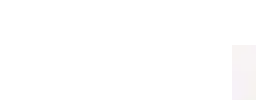

.

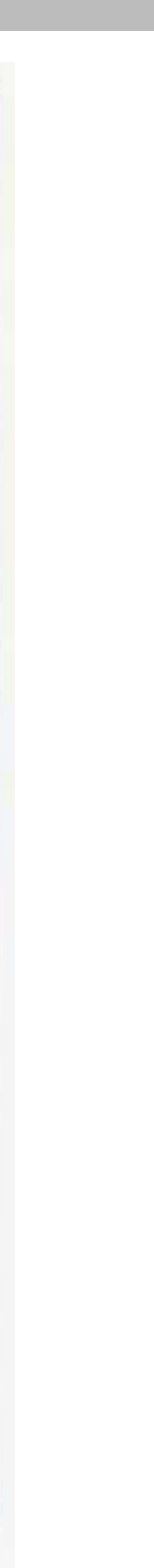

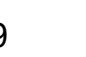

.




\title{
Mémoire
}

L'idéologie aliéniste du Dr Morel : christianisme social et médecine sociale, milieu et dégénérescence, psychiatrie et régénération.

Partie II

Doctor Morel's alienist ideology: social christianity and social medicine, environment and degeneration, psychiatry and regeneration.

\section{Part II}

\section{F. Carbonel}

Auteur correspondant : Frédéric Carbonel, Dr en histoire de l'université de Rouen, Faculté des lettres, 76821 Mont-Saint-Aignan Cedex, France

Adresse email : carbonel@ netcourrier.com

Tel perso : 0678515471

Texte reçu le 15 juin 2009 ; accepté le 7 novembre 2009

\section{Résumé}

Nommé à l'asile Saint-Yon en 1856, le Dr Morel développe sa théorie des dégénérescences «comme une dégradation originelle de la nature humaine ». Le « dégénéré » représente alors un danger biologique à combattre, aboutissant à la stérilité complète à la quatrième génération. Selon lui, seul le renforcement de la «Loi morale », centrée sur une médecine chrétienne sociale, permettra de «régénérer» la société afin d'extirper les « dégénérés » de leurs «milieux pathogènes ».

Mots clés : Christianisme social ; Loi morale ; Médecine sociale ; Milieu ; Morel

\begin{abstract}
Named at Saint-Yon asylum in 1856, Doctor Morel develops his theory of degeneration "like an original degradation of the human nature". "Degenerated" then represents a biological danger to fight leading to complete sterility with the $4^{\text {th }}$ generation. According to Doctor Morel only the reinforcement of the "moral Law" centered on a Christian-social medicine will make it possible "to regenerate" the society in order to extirpate "degenerated" of their "pathogenic environments".
\end{abstract}


Keywords: Christian-social medicine; Environment; Moral law; Morel; Social medicine

\section{Médecine sociale/Christianisme social, question sociale et dégénérescence}

Pour le chrétien social Morel, monogéniste et fixiste, la Bible et la Genèse étaient à l'origine de toute réflexion. La dégénérescence était une « dégradation originelle de la nature humaine ». Elle était « une déviation maladive d'un type primitif ». La fonction sociale de l'aliéniste consistait à combattre les causes de la destruction et de l'abâtardissement de l'espèce humaine car elles n'existaient pas au moment de la création. La recherche aliéniste devait se concentrer, selon le médecin de Saint-Yon, sur le «mode de production », la « classification », la «prophylaxie », l'« hygiène », et le «traitement» des êtres dégénérés. La théorie des dégénérescences faisait lien entre le christianisme social, la «question sociale » et la médecine [4,8]. De plus, le Traité des dégénérescences constituait une véritable enquête sur les conditions de vie et de travail de la classe ouvrière, qui s'étaient sensiblement détériorées depuis les années 1840. Pour l'aliéniste de Saint-Yon, la «question sociale et économique » bouleversait autant la constitution physique des individus que leurs mœurs. Il fallait en détecter tous les dangers [5,10].

\section{2. « Génération », « dégénérescence », « régénération » [1,3] (schéma 1)}

Dans la nouvelle société industrielle, le «dégénéré » devait être perçu, pour ses actions nuisibles, comme un coût selon plusieurs points de vue : un coût social pour les dépenses institutionnelles indirectes qu'il engendrait et les multiples mesures sociales qu'il fallait prendre à son encontre (notamment avec les dépenses des asiles pour aliénés, des maisons de sûreté et de sécurité). Mais le «dégénéré » représentait surtout un danger «biologique ». En effet, par son intermédiaire, de nombreuses conséquences néfastes pouvaient être introduites au sein même de toute la population. Ainsi, Morel était persuadé d'avoir mis en évidence une « loi des quatre générations » qui installait la « stérilité », produit de dégénérescences accumulées. Cette loi de «l'hérédité progressive » fut réévaluée par l'aliéniste Motet à la Société Médico-Psychologique en 1874. Ce constat d'une dégradation nette de l'organisation d'une extrémité à l'autre de l'espèce humaine pouvait poser de grands problèmes au sein des milieux populaires. Pire, cette notion de la transmission verticale de la dégénérescence de l'individu à son ascendant, hérédité accumulée et prédisposante (ou 
diathèse), se croisait, pour Morel, irrémédiablement avec celle de la transmission horizontale des individus à l'espèce. Une véritable contagion se propageait. Une «malaria physique et morale » prenait corps au sein de la société. Elle devenait un obstacle majeur au progrès social, dans le temps, de génération en génération, mais aussi dans l'espace, des classes les plus populaires à toute la société. Au cœur du problème de la «génération », le Dr Morel plaçait le thème de la «régénération » issu de la Révolution Française, mais dans une tout autre perspective [17]. Selon l'aliéniste, le paradigme des dégénérescences ne prenait tout son sens qu'en rapport avec celui de "régénération», puis de «génération ». L' "histoire naturelle » des dégénérés mettait au cœur de la problématique sociale l'expertise des généalogies. La «science médicale» se muait en «science sociale». Elle s'inspirait aussi d'une vision « organiciste» de l'« organisation sociale ». Avec Morel, la médecine mentale « convie à cette œuvre de régénération tous ceux auxquels sont confiés le bien-être et les destinées des populations, tous ceux qui possèdent les moyens de réaliser les projets d'amélioration que la science médicale soumet à leur examen ». «Génération/Régénération : les deux thèmes sont étroitement associés, dans toutes leurs connotations biologiques, psychologiques, morales, religieuses et messianiques » [13]. Selon le médecin de Saint-Yon, ce tableau dynamique permettait de comprendre la société à un moment donné : «Un faisceau de classes d'âge, un ensemble d'hommes et de femmes dont les idées, les sentiments et les manières de vivre sont les mêmes, et qui se présentent dans les mêmes conditions physiques, intellectuelles et morales aux faits et événements majeurs qui affectent la société dont ils sont un des éléments. » Comme le soulignera Motet : «Il était impossible que, conçue dans un esprit aussi large, cette œuvre magistrale ne touchât pas par plus d'un point à l'anthropologie ; aussi, l'influence des races y est-elle étudiée avec le plus grand soin et avec des développements considérables. Mais ce qu'il importe pour nous de faire ressortir, c'est l'action des grands modificateurs généraux, agissant soit séparément, soit simultanément sur des masses d'individus soumis aux mêmes influences, et s'accusant dans leurs effets, par des traits de plus en plus profonds, par le fait de l'hérédité de génération en génération. » Ainsi, la «folie », par exemple, se transférait de génération en génération tout comme la «syphilis héréditaire », tel un châtiment différé, mêlant accidents physiques et moraux, irritabilités nerveuses et troubles de la fonction reproductive, jusqu'à l'« abâtardissement ». Le Dr Morel classait les différents types $d$ ' «hérédité pathologique» selon son avancée dans les générations : première génération (la nervosité, l'irritabilité, la violence...), deuxième génération (les déficiences nerveuses graves : l'épilepsie, l'hystérie, l'hypocondrie...), troisième génération (les dispositions innées à la folie et la perversité, les instincts naturels 
mauvais...), enfin, quatrième génération (l'idiotie ou l'imbécillité complète accompagnée de stérilité). La progressivité héréditaire expliquait l'accentuation des troubles pathologiques chez les dégénérés. Elle aboutissait à la stérilité complète dès la quatrième génération. C'était le stade ultime de la déchéance complète de l'espèce humaine. Morel caractérisait aussi la démence précoce reprise quelques années plus tard par le Dr Kraepelin (1856-1926). Elle était une catégorie de la « folie héréditaire » : une «transition précoce à l'idiotisme » [2].

\section{Schéma 1 : La théorie des dégénérescences du Dr Morel, ou une légitimation théorique du rôle social des psychiatres sous le second Empire}

Postulat/Dogme créationniste chrétien (issu de la Genèse). $\square$

$\square+>$ Déviations maladives du «type » originel dues aux mauvaises conduites humaines et à l'organisation sociale qui les accompagne.

$\square+>$ Multiples dégradations/transformations de la nature originelle de l'espèce humaine.

$\square+>$ Dégénérescences physiques et morales. Apparition des différentes pathologies physiologiques, intellectuelles et morales.

$$
\Downarrow \Downarrow
$$

Fonction de la «Médecine sociale » : étudier les causes et les différents aspects des processus dégénératifs, en faire une typologie et les expliquer.

(par exemple : définir un « Milieu » propice ou non à la « dégénérescence »)

$\square$ Objectif de la psychiatrie : définir la « loi morale ».

$\square+>$ Faire le plan d'une Hygiène physique et morale pour favoriser les éléments régénérateurs.

$\square+>$ Élaborer un programme de Régénération/Remoralisation des masses afin d'améliorer les destinées des générations futures.

\section{$\Downarrow \Downarrow$}

Revenir à... (Régénérer l'espèce humaine).

Homme originel : « Type » normal de l'Humanité.

Postulat/Dogme créationniste chrétien. 


\section{Conception morélienne de la dégénérescence $[7,12,14,19]$}

Morel définissait des «variétés de dégénérés reconnaissables. Les «aliénésdégénérés » pouvaient être classés $\left(2^{\mathrm{e}}\right.$ classe, $3^{\mathrm{e}}$ classe...) selon une certaine typologie, de même qu'il existait des types, des variétés et une pluralité des races humaines. Cependant, Morel croyait à une unicité originelle du «genre humain » créé par Dieu. Néanmoins, les caractères acquis, de l'ordre physique et de l'ordre moral, des «dégénérés » permettaient de déterminer la catégorie des variétés maladives de l'espèce humaine auxquels ils appartenaient. Le Dr Morel présentait à l'appui de ses opinions des spécimens d'anthropologie pathologique. Il montrait par exemple qu'à partir d'un individu « dégénéré », il était possible non seulement de porter un diagnostic certain, mais de faire remonter l'individu à l'origine des causes qui avaient amené son «état dégénératif ». Il développait aussi l'idée que tous les "êtres dégénérés » avaient des caractères communs qui les rattachaient à la même famille, avec des caractères distinctifs qui les faisaient entrer dans tel ou tel genre de ces « variétés maladives ». Ainsi, le médecin de Saint-Yon énonçait les critères pris en considération pour ses typologies. Parmi eux, les stigmates physiques étaient prépondérants : la forme de la tête et des oreilles, la petitesse et le rabougrissement de la taille, le développement tardif de la puberté, la stérilité et les infirmités humaines. Mais Morel différenciait les déviations de «l'ordre physique» de celles de «l'ordre intellectuel et moral», comme les tempéraments instinctifs jugés mauvais. Ces différences de classifications n'étaient pas sans rappeler la querelle sur la prédominance des « causes morales » dans l'émergence de la «folie » à l'époque de Parchappe. Le Dr Morel avait pour fil directeur ce «système biologique» et cet «organicisme » (schémas 2 et 3 ). L'ancien Saint-Simonien Auguste Comte (1798-1857) l'avait bien décrit, en plaçant au sein des interrogations sur le progrès des sociétés humaines cette «loi lamarckienne de modification morphologique par l'habitude et [...] l'hérédité des modifications acquises ». Auguste Comte s'était montré favorable au rôle que devaient jouer la science et les savants afin de « régénérer » la société [17].

\section{Schéma 2 : Les principes de la « loi morale» vecteurs de la Régénération.}

Les principes de la «Loi Morale » vecteurs de la «Régénération».

Dégénérescences $=>$ Médecine Sociale et Loi Morale $=>$ Régénération 


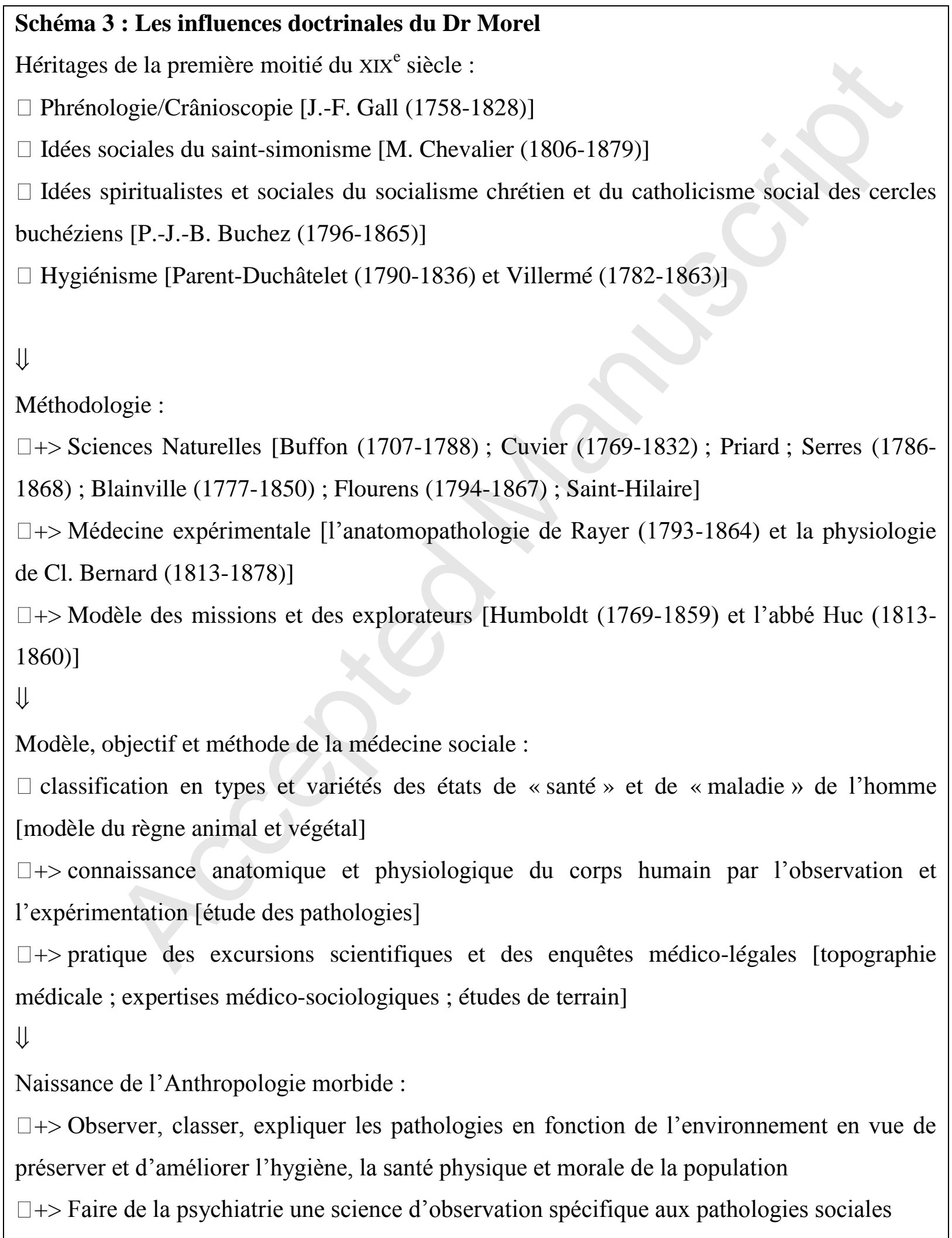




\section{Médecine mentale et défense de la « Loi morale »}

La « régénération », selon Morel, était reliée à la «Loi morale », précepte du comte de Saint-Simon, publié dans le Nouveau Christianisme : «Ces chefs de l'Église primitive ont fait le meilleur de tous les livres qui aient jamais été publiés, le catéchisme primitif, dans lequel ils ont partagé les actions des hommes en deux classes, les bonnes et les mauvaises, c'est-àdire celles qui sont conformes au principe fondamentale de la morale divine, et celles qui sont contraires à ce principe. » La référence à la « Loi morale » sera omniprésente dans l'œuvre du Dr Morel. Elle rejoignait la vision d'un ordre social identique à l'ordre bonapartiste. Proche des socialistes chrétiens, Morel avait trouvé ses premières justifications idéologiques chez Buchez surtout. Par la suite, il devait davantage s'inspirer de la philosophie ultraconservatrice de Louis de Bonald (1745-1840), déjà présente chez Buchez dans le volume II de son Essai d'un traité complet de philosophie du point de vue du catholicisme et du progrès, notamment dans sa partie «Doctrine de M. De Bonald» et dans le chapitre de la «Prééxistence de la morale à la société ».

\section{La « loi morale » préexiste à la société : le Dr Morel, lecteur de Bonald et Volney}

Morel «s'inspire de la philosophie conservatrice de L. de Bonald qui croyait à la dégénérescence sous l'effet des vices et des révoltes, d'une race humaine à l'origine modelée sur l'exemple divin. Il se situe donc à l'opposé de tout évolutionnisme (L'Origine des espèces ne paraîtra qu'en 1859)» de Darwin [15,16]. Morel avait certainement lu Bonald comme Buchez: «La morale est la seule des connaissances humaines qui satisfasse complètement aux conditions que nous avons établies précédemment comme constitutives à toutes choses humaines ; à la société car il n'y a point de société, ni même de langage possible sans morale ; au dogme et à la science, car c'est de la morale qu'émanent le dogme et la science. » Selon Morel, la primauté du religieux avait pour conséquence la primauté de l'ordre naturel du Créateur car pour Bonald comme pour le médecin de Saint-Yon, chaque individu devait se soumettre à la «loi morale ». L'individu n'existait que par la société dans laquelle il était intégré. Ainsi, la vision du Dr Morel s'inscrivait aussi dans une filiation avec les théories « organicistes » sociales du réactionnaire Bonald et du naturaliste normand Blainville. La «théorie des dégénérescences » s'inscrivait dans un «ordre moral », même s'il pouvait se 
distinguer de celui de Saint-Simon ou d'Auguste Comte. Bonald, auteur de la Législation primitive considérée dans les derniers temps par les seules lumières de la Raison (1817) et d'un Essai analytique sur les lois naturelles de l'ordre social (1836), pensait que le savant ne pouvait traiter de la société sans parler de l'homme, ni parler de l'homme sans remonter à Dieu. De même, le Dr Morel était très attaché à la défense des valeurs familiales traditionnelles. Il concevait la société en termes de devoirs des générations les unes envers les autres. La famille et l'autorité paternelle étaient le fondement de l'ordre social et politique. Elles étaient aussi des « vertus domestiques » nécessaires à la défense des valeurs bourgeoises telles que définies par l'idéologue Volney (1757-1820). Idéologue, membre de la Société des observateurs de l'homme, Volney avait été l'auteur en 1795 de Questions de statistiques à l'usage des voyageurs et d'un catéchisme du citoyen français paru en 1793. Il y avait décrit la «loi naturelle» et les «principes physiques de la morale, déduits de l'organisation de l'homme et de l'univers ». «Famille» et «Autorité» organisaient la vie sociale. Ces «hiérarchies naturelles » devaient être respectées. Or, ce traditionalisme politique rejoignait celui du christianisme social : un vaste rejet des mutations du monde moderne accusé de corruption et tournant le dos à la volonté divine. Ces idées correspondaient aussi au contexte social survenu après les événements de 1848. Le retour aux «valeurs » pouvait être une attente des couches populaires les plus démunies et brutalement confrontées aux méfaits de l'industrialisation. Dans cette perspective, le Dr Morel articulait la «théorie des dégénérescences » avec la régulation et la norme dans la cité ouvrière. La «sexualité » et la transmissibilité de la «tare » dite héréditaire étaient les principaux aspects voués à être régulés par la discipline aliéniste et la «loi morale». Comme Buchez, l'aliéniste Morel s'opposait à l'atomisme social que les principes de 1789 semblaient avoir renforcé. Le médecin de Saint-Yon combattait la disparition des maillons sociaux et des repères ancestraux car les valeurs libérales poussaient, pour l'aliéniste, à l'instabilité et à l'insécurité. C'est pourquoi la pensée sociale de Morel était très proche du traditionalisme bonaldien. Sa vision rencontrait celle du très catholique social Buchez qui avait écrit dans son Histoire de la nationalité française, paru en 1859, deux ans après le Traité des dégénérescences: «L'homme peu perfectionner son organisme, ou accroître la puissance dans des directions diverses ; mais il peut aussi l'amoindrir et la détériorer. Ces résultats, bons ou mauvais, sont transmissibles aux enfants. Le mal comme le bien sont organiquement héréditaires ; mais Dieu a voulu que le mal conclût rapidement à dégénérescence, et que la race où il s'est trop fortement implanté devînt assez promptement stérile. » L'aliéniste Buchez rejoignait Morel sur ce constat : l' «être dégénéré, s’il est abandonné à lui-même, tombe dans une dégradation 
progressive. Il devient [...] non seulement incapable de former dans l'humanité la chaîne de transmissibilité d'un progrès, mais il est encore l'obstacle le plus général à ce progrès, par son contact avec la partie saine de la population. La durée de son existence enfin est limitée comme celle de toutes les monstruosités ». Selon Morel, les dégénérés n'étaient que «les représentants de l'hérédité de mauvaise nature accumulée dans leur personne ».

\section{Dégénérescences, critique de l'industrialisation et anti-modernisme}

Les idées de Morel se situaient à l'opposé de celles des catholiques ultralibéraux car elles voulaient, tout en préservant l'ordre établi, améliorer directement la condition ouvrière. Elles se situaient dans une continuité de préoccupation avec celles des évêques des grandes régions industrielles, notamment celle de Rouen. Elles compatissaient au paupérisme urbain. Le Dr Morel dénonçait directement les abus du travail dans les ateliers de textile de la SeineInférieure, notamment celui des enfants. Il rejoignait les préoccupations et la sensibilité sociale des bourgeois et des patrons de Rouen. En effet, les catégories supérieures rouennaises, notamment catholiques, étaient horrifiées par les conditions du travail des enfants en Normandie. La bourgeoisie rouennaise était profondément marquée par la réflexion sociale de l'Église catholique dans le diocèse de Rouen. Elle s'était singulièrement opposée au concept ultralibéral de la «liberté sans frein » en diffusant une «masse d'écrits sociaux », notamment dans les ateliers des patrons catholiques. En Seine-Inférieure, la configuration des fabriques d'indiennes pouvait favoriser le développement d'une politique sociale novatrice, menée par les patrons sociaux catholiques et les protestants de la région normande. Dans ce contexte, les activités du Dr Morel pouvaient servir d'exemple pour la philanthropie religieuse essentiellement catholique. Dans les années 1850, Frédéric Le Play (1806-1882), le fondateur de la sociologie chrétienne, avait fait paraître sa vaste enquête sur Les ouvriers européens. Simultanément, en 1856, le Traité des dégénérescences physiques, intellectuelles et morales de l'espèce humaine et des causes qui produisent ces variétés maladives marquait le renforcement d'une psychiatrie catholique engagée fondée sur des modes de pensée identiques : le « Bien »/le « Mal », l'obsession du social et de la « santé morale » des familles. Ces valeurs étaient des bases essentielles de l'existence pour les chrétiens sociaux. 


\section{Tableau 1 : Les déterminismes du milieu et leurs conséquences sur les êtres humains selon l'aliéniste Morel}

Agents intoxicants, hygiène et climats

Poisons (alcool, chanvre, opium, tabac)

Mauvaise alimentation (ergotisme, pellagre, scrofules, nourriture frelatée, carences nutritionnelles)

Climats (effluves palustres, marais, terrains insalubres)

Maladies courantes (goitre, crétinisme, paludisme)

Toxiques industriels

Dispositions organiques maladives

Infirmités morales et physiques

Affections physiques et nerveuses spécifiques

\section{Propension au crime}

Immoralité des parents

Promiscuité des sexes

Éducation vicieuse des enfants

Schéma 4 : La détection des dégénérés et le problème de l'« hérédité progressive » selon le Dr Morel 
Détection des dégénérés et problème de l' « hérédité progressive » selon le Dr Morel :

Problème de l'hérédité => territoires et « circonstances » $=>$ populations et sociétés

\section{Milieux pathogènes, géographie médicale et « Anthropologie morbide » : influences} des « sciences naturelles » dans la théorie des dégénérescences $[6,9,11]$

Installé à Rouen en 1856, Morel allait appliquer les techniques de la «Médecine sociale » à la Seine-Inférieure. Il pouvait aussi s'inspirer du modèle de la «Géographie médicale» et des «Sciences Naturelles ». Le Dr Morel avait défini l'importance de sa mission à l'asile Saint-Yon : «Nous ne sommes plus en effet en face d'un homme isolé, mais en présence d'une société, et la puissance des moyens d'action devra être en rapport avec l'importance du but » avertissait-il. En janvier 1858, les Annales Médico-Psychologiques insistaient sur l'intérêt de la «Géographie médicale » pour l'ensemble de la communauté aliéniste. En outre, ils indiquaient que les naturalistes avaient donné l'exemple aux aliénistes de la marche à suivre dans l'étude des caractères humains (tableau 1). Le Traité des dégénérescences avait l'ambition d'être un véritable recueil de savoir anthropologique, géographique, et ethnologique. Le Dr Morel reprenait le paradigme de Gall : diagnostiquer «l'état de santé et de maladie chez l'homme », de la même manière que «l'état de santé et de maladie chez tous les êtres créés du règne animal et du règne végétal » (schéma 4). Il s'appuyait aussi sur les travaux physiologiques de Claude Bernard et l'anatomopathologie de Rayer. Élève de Blainville, lecteur assidu de Cuvier, Buffon, Geoffroy de Saint-Hilaire, Serres, Flourens et Lamarck, Morel s'inspirait directement des théories issues des « Sciences Naturelles »: le fixisme et le transformisme. Dans son Discours sur les révolutions de la surface du globe, le naturaliste fixiste Cuvier s'était demandé en 1825 : «Pourquoi les races actuelles, me dira-t-on, ne seraient-elles pas des modifications de ces races anciennes, (que l'on trouve parmi les fossiles), modifications qui auraient été produites par les circonstances locales et le changement de climat, et portées à cette extrême différence par la succession des années » [9]. Le Dr Morel considérait «l'anthropologie morbide » comme une branche de la biologie générale. L'homme était soumis aux mêmes lois de santé et de maladie que chez tous les êtres vivants. «Dégradation et simplification de l'organisation d'une extrémité à l'autre de la chaîne animale, en procédant du plus composé vers le plus simple » avait expliqué Lamarck dans sa Philosophie zoologique en 1809. Néanmoins, le Dr Morel raisonnait à l'inverse de Lamarck: des lois faisaient se transformer l'homme originel vers les types les plus variés. Cependant le Traité des dégénérescences portait la marque du transformisme lamarckien qui 
faisait de l'évolution des espèces, et de son explication par des causes matérielles, un principe de base. Morel avait parfaitement compris le raisonnement général : «l'action du milieu (Lamarck dit des «circonstances ») agissant sur les organismes » et «déterminant les habitudes qui à la longue modifient leur morphologie et se transmettent à leur descendance » [9]. La détermination d'un «milieu» propice à la diffusion des dégénérescences était fondamentale pour la compréhension du paradigme élaboré par Morel. L'influence de Geoffroy de St-Hilaire était très perceptible pour définir l'hérédité des caractères somatiques acquis dans la théorie morélienne. Geoffroy de Saint-Hilaire avait scruté les monstruosités dans son Histoire des anomalies de l'organisation ou Traité de Tératologie paru entre 1832 et 1836. Selon Morel, les déviations maladives de l'espèce humaine étaient à la fois la conséquence des conditions pathogènes du «milieu » mais aussi des «caractères acquis » à la longue en son sein. Ainsi, les pathologies physico-mentales se transmettaient héréditairement tout en se modifiant de génération en génération. Ainsi, les anomalies physiques autant que psychiques en étaient le reflet. De l'ensemble de ses observations, le Dr Morel en avait déduit que «la théorie des dégénérescences dans l'espèce humaine [...] amenait la décadence progressive de telle ou telle famille, et parfois même de telle ou telle race ».

Le Dr Morel voulait mener une véritable «anthropologie de la Seine-Inférieure ». Il avait toujours à l'esprit ses préoccupations initiales exposées en 1848. Nous les avons développées précédemment. Une meilleure compréhension des «dégénérescences » devait préserver les destinées humaines futures de ses effets néfastes. De ce point de vue, la SeineInférieure était, pour Morel, «un nouveau milieu bien plus fertile en explorations scientifiques » que ceux qu'il avait pu fréquenter auparavant. En effet, la Seine-Inférieure était un des départements français les plus industrialisé. Cependant, «j'examine la question en médecin et non pas en économiste » expliquait Morel. L'anthropologie envisagée par le Dr Morel était sensiblement imprégnée par le système d'intelligibilité néo-hippocratique sur les «airs, les eaux et les lieux » [18]. En effet, le bon diagnostic médical d'une population passait nécessairement par la description naturelle de la «contrée » d'existence, la nature et la disposition physique du sol, la qualité de l'air et des eaux, autant de critères « typiques » de la «Géographie médicale ». L'étude des pathologies humaines devait s'accompagner d'une description fine des «circonstances » et des habitudes de vie : les structures de la population active, les conditions d'habitation, les aliments, les boissons et les mœurs devaient être passés au crible par les aliénistes. Morel présentait ainsi l'intérêt principal de son enquête : «Il m'est impossible désormais de séparer l'étude de la pathologie des maladies mentales de celle des causes qui produisent les dégénérescences fixes et permanentes, dont la présence, au milieu de 
la partie saine de la population, est un sujet de danger incessant. » Il voulait compléter son exposé des genres de vie par celui de l'environnement en se fixant trois objectifs : faire une étude des «causes dégénératrices », réaliser une classification des variétés maladives, les expliquer par leur «solidarité » et leur formation à l'intérieur même de «l'espèce humaine ». La perspective globalisante de «l'œuvre morélienne» permettait d'élaborer une grille de lecture hygiéniste des pathologies mentales. Cette volonté explicative était aussi celle des chrétiens-sociaux. Elle rejoignait celle des saint-simoniens et des bonapartistes. De plus, l'analyse de Morel s'affiliait aux enquêtes d'investigation présociologique des hygiénistes Parent-Duchatelet (1790-1836) et Villermé (1788-1863).

En outre, Morel ne négligeait pas d'évoquer l'intérêt qu'avaient les administrateurs de la Seine-Inférieure à étudier ces documents «utiles sur les causes de l'augmentation des aliénés et sur les moyens hygiéniques et prophylactiques les plus propres à prévenir une aussi grande infirmité ». Dans ce but, Morel avait distingué les différents types de milieux à risques : les milieux méphitiques, les quartiers insalubres, les cités minières (non présentes en Seine-Inférieure) et de fabriques (très présentes en Normandie), et, enfin, les microenvironnements pathogènes où s'entassaient les victimes d'intoxications comme ceux des cabarets avec ses ouvriers ivrognes. Ces derniers entraînaient, par mimétisme, les aubergistes à devenir à leur tour alcooliques !

\section{Le Dr Morel, enquêteur social, à la recherche de la définition du « milieu pathogène »}

Morel semblait ne rien laisser au hasard. Il notait l'importance des famines, des professions dangereuses, de la misère sociale et économique, de l'état moral des populations, de l'hérédité des handicaps et des infirmités, de la fréquence des nourritures carencées et avariées. Il analysait les mauvaises influences hygiéniques de la constitution des sols comme celles des terrains marécageux, d'où pouvaient surgir le paludisme et les fièvres intermittentes. « La progression dans la composition de l'organisation subit, çà et là, dans la série générale des animaux, des anomalies opérées par l'influence des circonstances d'habitation et par celle des habitudes contractées » avait expliqué Lamarck. La vie des « dégénérés » et leurs caractéristiques organiques étaient identiques à celles des animaux. C'est alors que plusieurs causes mixtes pouvaient s'entremêler pour qualifier un «milieu pathogène ».

\section{Conclusion}


La «dégénérescence » se percevait surtout grâce à la prolifération des «stigmates objectifs » indiquant, à travers la dysharmonie du corps et la désorganisation du système nerveux les «désordres de la conduite » et des «milieux»: «personne n'avait affirmé que certaines maladies, certaines intoxications, certaines habitudes chez les parents eussent la puissance de créer, chez les enfants, un état véritablement consécutif, un état organique spécial, indéfiniment transmissible jusqu'à extinction de la race, si rien n'était fait pour le changer ». Les conditions d'existences de certaines catégories de la population française, notamment en Seine-Inférieure, étaient à ce point incroyables que le nouveau médecin de Saint-Yon faisait des voyageurs et des explorateurs un point d'ancrage essentiel de sa nouvelle méthode. Ainsi, Morel prenait pour exemple le missionnaire français Régis Huc (1813-1860) qui avait rapporté de ses missions en terre jaune ses commentaires sur l'usage de l'opium, la constitution géologique des sols et son lien avec les goitreux et les crétins de l'Empire chinois et du Tibet! Le Dr Morel utilisait non seulement les enquêtes de ces prédécesseurs : le Tableau de l'état physique et moral des ouvriers dans les manufactures de coton, de laine et de soie de Villermé publié en 1840 (des recherches menées entre 1835 et 1837), mais aussi les Études sur l'Angleterre de Léon Faucher (1803-1854) publiées en 1845, ainsi que les Lettres sur l'organisation du travail, et les Études sur les principales causes de la misère et sur les moyens propres pour y remédier, de Michel Chevalier (18061879) parues en 1848. Par ailleurs, Morel tenait compte des recherches les plus récentes en anthropologie sur la consanguinité des colons portugais de Macao, la vie des peuplades d'Amérique et des Esquimaux, ainsi que les travaux de Magnus Huss sur l'alcoolisme en Suède. Magnus Huss avait reçu le prix de Vertu Montyon de l'Académie des Sciences en 1854. Les préoccupations sur l'essor des «milieux pathogènes » rejoignaient celles des sociétés savantes de la Seine-Inférieure proches des milieux bourgeois et industriels comme la Société libre d'émulation du commerce et de l'industrie de la Seine-Inférieure. Ainsi, au concours que cette dernière avait lancé en 1869, un des ouvrages retenu était justement celui intitulé Un voyage en zig-zag chez nos ennemis. Entretiens populaires sur l'Hygiène. La brochure se voulait didactique et scientifique. Sa Table des matières se terminait par les avantages d'une santé robuste après avoir évoqué tous les dangers pour l'organisme des agents intoxicants : «malpropreté », «dangers des préparations de plomb », « de l'air altéré, vicié », « air confiné, miasmes », « ennemis de la digestion », et «alimentation mal digérée ». Tous ces périls avaient des conséquences très importantes sur l'état physique et moral des habitants de la Seine-Inférieure. En expert hygiéniste, le Dr Morel plaçait la question de 
l'hérédité dans le cadre conceptuel de son époque soit des phénomènes physiologiques et sociaux posés à un moment donné à une certaine catégorie de la population sur un territoire déterminé.

L'étude de «l'homme physique », non isolé de celle de «l'homme moral», concourait à la naissance des «sciences de l'homme» autant qu'à la consolidation des «sciences morales » et de «gouvernement ». Au tournant des années 1850-1860, le Dr Morel exposait ainsi au préfet de la Seine-Inférieure son programme d'anthropologie psychiatrique. Il passait par le repérage sur le terrain des populations les plus frappées par le paupérisme : « Il est nécessaire, dans bien des cas, de pénétrer dans l'intérieur des familles, de voir de près la manière de vivre des habitants d'une localité, de se mettre au courant de leur hygiène physique et morale [...] Je ne crois pas que l'on puisse parvenir autrement à faire la statistique morale de cet important département» écrivait Morel. La «Géographie médicale » et la question de l'«héréditarisme» en Seine-Inférieure ne pouvaient s'expliquer que par les « circonstances» des bouleversements économiques et sociaux intrinsèques: le fort prolétariat industriel aggloméré dans la région rouennaise. Lors de ces premières visites dans le quartier des jeunes délinquants de la prison de Gaillon dans l'Eure, le Dr Morel avait pu constater l'origine géographique et sociale des jeunes prisonniers. La plupart des délinquants étaient issus du Pollet, un quartier de marins excessivement pauvres de Dieppe. «Leurs instincts malfaisants" s'accompagnaient des «formes vicieuses de leurs têtes», de «l'abaissement de l'intelligence», des «arrêts du développement» et « de la stérilité absolue ». Selon le Dr Morel, ces «jeunes» formaient «des types sociaux de dégénérescence » provoqués par «la constitution vicieuse des parents ».

\section{Conflits d'intérêts : à compléter par l'auteur}

\section{Sources}

Annales Médico-Psychologiques. De l'hérédité morbide progressive, rapport de J.P. Falret, $1867 ; 10$.

Benedict Augustus Morel (1809-1873). In: Pioneers in Mental Hygiene, Mental Hygiene 1930;14:462-3.

Buchez Ph. (1857), Rapport fait à la Société Médico-Psychologique sur le Traité des dégénérescences physiques, intellectuelles et morales de l'espèce humaine et des causes qui les produisent par le Dr B.-A. Morel. Ann Méd Psychol 1857;3.

La Revue Nationale 1848;2:441-2 et 1848;7:504-5. 
Morel BA. (1857), Traité des dégénérescences physiques, intellectuelles et morales de l'espèce humaine et des causes qui produisent ces variétés maladives, Paris, Londres, New York, Baillière, 700 p. avec Atlas de XII planches, Paris, Londres, New York, Baillière, 23p.

Morel BA. Études cliniques. Traité théorique et pratique des maladies mentales dans leur nature, leur traitement et dans leur rapport avec la médecine légale des aliénés, Nancy, Grimblot et Vue Raybois. Paris: Masson, Baillière; 1852, 2 tomes.

Morel BA. Traité des maladies mentales. Paris: Masson; 1860. 866p.

Morel BA. Questions sur diverses branches des sciences médicales. Thèse de médecine ; 6 août 1839. 34p.

Morel BA. Des caractères de l'hérédité dans les affections nerveuses. Archives générales de médecine 1859, p.257-83.

Morel BA. De la folie héréditaire. Rapport médico-légal sur un individu qui comptait des aliénés dans son ascendance paternelle et maternelle et qui a été condamné pour outrages aux moeurs précédé de considérations sur les actes immoraux des aliénés. Gazette hebdomadaire de médecine et de chirurgie 1862. 29p.

Morel BA. De l'hérédité morbide progressive ou des types dissemblables et disparates dans la famille. Archives générales de médecine : avril 1867. 51p.

Morel BA. De la formation de type dans les variétés dégénérées. Précis analytique des travaux de l'Académie de Rouen : 1863/1864. p. 106-7.

Morel BA. De l'hérédité morbide progressive ou des types dissemblables et disparates dans la famille. Ann Méd Psychol 1869;1:329-30.

Morel (1809-1873), médecin de l'asile Saint-Yon. La Revue Médicale de la Normandie 1900;3:58-61.

Morel BA. In: Paris : Dictionnaire biographique illustré de la Seine-Inférieure, $2^{\mathrm{e}}$ éd ; 1908. p.819.

Mort de monsieur le Dr Morel. Union Médicale de la Seine-Inférieure ; 15 avril 1873. p.4-72.

Motet A. Éloge de Morel. Ann Méd Psychol 1874;12:82-108.

\section{Références}

[1] Bénichou C. Enquête et réflexions sur l'introduction des termes «dégénére(r)», « dégénération », « dégénérescence » dans les dictionnaires et encyclopédies scientifiques françaises à partir du XVII ${ }^{\mathrm{e}}$ siècle. Documents pour l'histoire du vocabulaire scientifique $1983 ; 5: 1-62$. 
[2] Bonfils B. Histoire du concept de démence précoce en France. Paris: thèse de médecine; 1979. 73p.

[3] Clervoy P, Corcos M. Bénédict Augustin Morel (1809-1873): lutter contre la dégénérescence : pour régénérer quoi ? Perspectives Psychiatriques 2001;40:171-98.

[4] Coffin JC. L'hérédité et la question sociale (fin XIX ${ }^{\mathrm{e}}$ siècle). In: Gueslin A, Stiker HJ (Dir.) Handicaps, pauvreté et exclusion dans la France du XIX ${ }^{\mathrm{e}}$ siècle. Paris : Ed. de l'Atelier ; 2003. p.219-60.

[5] Coffin JC. La médecine mentale et la Révolution de 1848 : la création de la Société Médico-Psychologique. Revue d'Histoire du XIX ${ }^{\mathrm{e}}$ siècle 1998;16:83-94.

[6] Coffin JC. Heredity, Milieu and Sin: the Works of Bénédict-Augustin Morel (1809-1873). In: Rheinberger HJ, Müller-Wille S (Dir.) The Cultural History of Heredity. Berlin: Max Planck Institute; 2003. p.153-64.

[7] Debrock M. De l'actualité de l'œuvre de Benedict Augustin Morel. Lille II: thèse de médecine; 1984.

[8] Dowbiggin I. Degeneration and hereditarianism in French mental medicine 1840-1890: psychiatric theory as ideological adaptation. In: The Anatomy of Madness: Essays in the History of Psychiatry, Bynum WF, Porter R, Schaperd M (Dir.). Londres: Tavistock, t.1, «People and Ideas »; 1985. p.188-231.

[9] Drouin JM, Lenay C. Théories de l'évolution. Une anthologie. Paris: Press Pocket; 1990.

[10] Friedlander R. BA Morel and the Theory of Degenerescence: The Introduction of Anthropology into Psychiatry. San Francisco: University of California, Ph.D. Thesis; 1973.

[11] Krams-Lifschiftz A. Dégénérescence et personne, migrations d'un concept au XIX siècle. In: Novaes S (Dir.) Biomédecine et devenir de la personne. Paris: Seuil; 1991. p.131-58.

[12] Martin Cl. La dégénérescence dans l'œuvre de B.-A. Morel et dans sa postérité. Thèse de $3^{\mathrm{e}}$ cycle, 2 vol ; 1983.

[13] Nora P. «La génération » dans «Partages de l'espace-temps ». Les lieux de mémoire 1997;2:2975-3015.

[14] Pick D. Faces of Degeneration. A European Disorder, 1848-1918. Cambridge: Cambridge University Press; 1989.

[15] Postel J. L'idée de dégénérescence en psychiatrie et l'introduction du darwinisme en France au XIX ${ }^{\mathrm{e}}$ siècle. Information psychiatrique 1976;52:855-6.

[16] Postel J. Morel BA. Encyclopaedia Universalis 1985. P. 2013

[17] Régénération et reconstruction sociale entre 1780 et 1848 . Recueil collectif, Université de Nice, Centre de Recherches d'histoire des idées. Paris: Vrin; 1978. 
[18] Vigarello G. La force de soi, la force des autres. XIX ${ }^{\mathrm{e}}$ siècle. In: Histoire des pratiques de santé. Le sain et le malsain depuis le Moyen Âge. Paris: Seuil; 1993 (notamment le chapitre II «L'invention de l'énergie -1- Le spectre des dégénérescences », p. 217-30, et le chapitre III «De l'hygiène des lieux à l'hygiène mentale », p. 251-83).

[19] Zaloszic A. Éléments d'une histoire de la théorie des dégénérescences dans la psychiatrie française. Strasbourg: thèse de médecine; 1975;107:238. 